\title{
915 MOLECULAR CHARACTERIZATION OF AXL IN SOLID TUMOR MALIGNANCIES USING REAL-WORLD DATA
}

${ }^{1}$ Han $\mathrm{Si}^{*}$, 'Maria Jure-Kunkel, 'Nora Pencheva, ' ${ }^{1}$ Steven Xu, ${ }^{1}$ Brandon Higgs, ${ }^{1}$ Kate Sasser, ${ }^{1}$ Hisham Hamadeh, ${ }^{2}$ Phaedra Agius, ${ }^{2}$ Kristina Grigaityte. ${ }^{1}$ Genmab Inc, Rockville, MD, USA;

${ }^{2}$ Tempus Labs, Inc, Chicago, IL, USA

Background The receptor tyrosine kinase AXL is aberrantly expressed in many cancer types and associated with epithelialto-mesenchymal transition (EMT), poor prognosis, and therapy resistance. To better understand the expression of this gene across specific disease subtypes, correlated pathways, and how certain therapies potentially modulate AXL expression, we investigated real-world clinical and molecular data across five solid tumor types before and after chemotherapy or immune checkpoint inhibitor (CPI) therapy.

Methods Whole transcriptome and exome sequencing were derived from patient tumor specimens obtained either prior to treatment or following chemotherapy or CPI therapies. RNA reads were mapped using STAR and data was normalized using transcripts per million. DNA reads were mapped using Novoalign and variants were called using Freebayes and Pindel. Clinical data was curated from multiple sources, QC'd and deidentified according to standard protocols. Five diseases were included: non-small cell lung cancer (NSCLC, $\mathrm{n}=1181$ ), ovarian cancer (OV, $\mathrm{n}=300)$, urothelial carcinoma (UC, $\mathrm{n}=140$ ), pancreatic ductal adenocarcinoma (PDAC, $\mathrm{n}=942$ ), and skin cutaneous melanoma (SKCM, n=157). PD-L1 positivity was defined as $\geq 1 \%$ tumor cells with PD-L1 immunohistochemical staining at any intensity.

Results AXL mRNA levels were highest in PDAC followed by NSCLC, SKCM, UC and OV. Within OV, AXL expression levels were higher in tumors pre-treated with chemotherapy relative to untreated. For other tumor types, chemotherapy or CPI pre-treated tumors had AXL mRNA levels comparable to untreated tumors. Copy number amplifications of AXL were rare across all tumor types $(<3 \%)$ and did not associate with mRNA expression. Distinct molecular subtypes in several cancers displayed relatively high AXL mRNA levels, including the mesenchymal subtype in OV and the stromal rich subtypes in PDAC. AXL levels also correlated with an EMT mRNA signature across all tumors $(\mathrm{rho}=0.67)$. Further, higher AXL expression was associated with PD-L1 positivity in NSCLC, UC and PDAC $(\mathrm{p}<0.01)$, but not OV where only a few tumors were PD-L1 positive.Oncogenic KRAS mutations were associated with higher AXL expression in NSCLC and PDAC $(p<0.001)$ and lower AXL expression in OV $(p=0.01)$. Loss of KDM6A, known to induce tumorigenesis in PDAC, was associated with higher AXL expression in PDAC $(\mathrm{p}<0.01)$. Loss-of-function mutations in ARID1A, previously implicated as CPI sensitizing, were associated with lower AXL mRNA levels in OV tumors $(\mathrm{p}<0.001)$.

Conclusions Analyses of real-world mRNA datasets showed that AXL was upregulated in specific tumor and treatment settings as well as in patient populations with specific mutations and disease subtypes. Findings here should be validated with independent datasets.

http://dx.doi.org/10.1136/jitc-2021-SITC2021.915 